\title{
ENTIRE POSITIVE SOLUTION TO THE SYSTEM OF NONLINEAR ELLIPTIC EQUATIONS
}

\author{
LINGYUN QIU AND MIAOXIN YAO
}

Received 8 November 2005; Revised 12 May 2006; Accepted 15 May 2006

The second-order nonlinear elliptic system $-\Delta u=f_{1}(x) u^{\alpha}+g_{1}(x) u^{-\beta}+h_{1}(x) u^{\gamma} P(v)$, $-\Delta v=f_{2}(x) v^{\alpha}+g_{2}(x) v^{-\beta}+h_{2}(x) v^{\gamma} P(u)$ with $0<\alpha, \beta, \gamma<1$, is considered in $\mathbb{R}^{N}$. Under suitable hypotheses on functions $f_{i}, g_{i}, h_{i}(i=1,2)$, and $P$, it is shown that this system possesses an entire positive solution $(u, v) \in \mathbb{C}_{\text {loc }}^{2, \theta}\left(\mathbb{R}^{N}\right) \times \mathbb{C}_{\text {loc }}^{2, \theta}\left(\mathbb{R}^{N}\right)(0<\theta<1)$ such that both $u$ and $v$ are bounded below and above by positive constant multiples of $|x|^{2-N}$ for all $|x| \geq 1$.

Copyright (c) 2006 L. Qiu and M. Yao. This is an open access article distributed under the Creative Commons Attribution License, which permits unrestricted use, distribution, and reproduction in any medium, provided the original work is properly cited.

\section{Introduction}

This paper is concerned with the second-order nonlinear elliptic system

$$
\begin{aligned}
& -\Delta u=f_{1}(x) u^{\alpha}+g_{1}(x) u^{-\beta}+h_{1}(x) u^{\gamma} P(v), \\
& -\Delta v=f_{2}(x) v^{\alpha}+g_{2}(x) v^{-\beta}+h_{2}(x) v^{\gamma} P(u),
\end{aligned} \quad x \in \mathbb{R}^{N}(N \geq 3),
$$

where $\Delta$ is the Laplacian operator, $0<\alpha, \beta, \gamma<1$ are constants, the functions $f_{i}, g_{i}, h_{i}(i=$ $1,2)$ are nonnegative and locally Hölder continuous with exponent $\theta \in(0,1)$ in $\mathbb{R}^{N}$, and $P: \mathbb{R}_{+} \rightarrow \overline{\mathbb{R}}_{+}$is a continuous differentiable function, where $\mathbb{R}_{+}=(0,+\infty), \overline{\mathbb{R}}_{+}=[0,+\infty)$.

We are interested in the study of the existence of entire positive solutions $(u(x), v(x))$ to (1.1) which satisfy the condition that each of its elements decays between two positive multiples of $|x|^{2-N}$ as $x$ tends to infinity. By an entire solution of (1.1) is meant a pair of functions $(u, v) \in \mathbb{C}_{\text {loc }}^{2, \theta}\left(\mathbb{R}^{N}\right) \times \mathbb{C}_{\text {loc }}^{2, \theta}\left(\mathbb{R}^{N}\right)$ which satisfies (1.1) at every point $x$ in $\mathbb{R}^{N}$.

The existence of entire positive solutions of the equation

$$
\Delta u+f(x, u)=0, \quad x \in \mathbb{R}^{N}, N \geq 3,
$$


has been proved under various hypotheses, see $[6,7,10-12,14,17]$. In Particular, for the generalized Emden-Fowler equation

$$
\Delta u+K(x) u^{\lambda}=0, \quad x \in \mathbb{R}^{N}, N \geq 3
$$

where $\lambda$ is a constant, and $K$ is a positive locally $\theta$-Hölder continuous function in $\mathbb{R}^{N}$, Fukagai [7] has proved for $\lambda \in(0,1)$ that if

$$
\int_{1}^{+\infty} s^{N-1-\lambda(N-2)} K^{*}(s) d s<+\infty, \quad K^{*}(s)=\max _{|x|=s} K(x)
$$

then there is an entire positive solution of (1.3) that is minimal, that is, bounded below and above, respectively, by a positive constant times $|x|^{2-N}$ as $x$ tends to infinity.

Equation (1.3) with $\lambda \in(0,1)$ is said to be of sublinear type; if $\lambda$ is negative, then (1.3) is said to be of singular type, and such equations arise from the boundary layer theory of viscous fluids, see $[3,13]$. In this paper, we focus on elliptic systems of mixed type.

It is well known that some reaction-diffusion equations have been investigated in connection with models of population dynamics $[2,5,9,15]$. To mention some, in [15], the equation $\partial u / \partial t-d \Delta u^{m}=f(x, u)$ is studied. For some mutualistic symbiosis population models of two species, it may be necessary to study equation systems such as

$$
\begin{aligned}
& \frac{\partial u}{\partial t}-d_{1} \Delta u^{m}=f_{1}(x) u^{\rho}+g_{1}(x) u^{\sigma}+h_{1}(x) u^{\mu} P(v), \\
& \frac{\partial v}{\partial t}-d_{2} \Delta v^{m}=f_{2}(x) v^{\rho}+g_{2}(x) v^{\sigma}+h_{2}(x) v^{\mu} P(u),
\end{aligned} \quad x \in \mathbb{R}^{N},
$$

where $0<\rho, \mu<m,-m<\sigma<0$, and $d_{1}, d_{2}>0$. Obviously, the positive equilibrium solutions to system (1.5) in $\mathbb{R}^{N}$ are corresponding to the entire positive solutions of a system in the form of (1.1).

Some existence results of elliptic system

$$
\begin{gathered}
\Delta u+F_{1}(x, u, v)=0, \\
\Delta v+F_{2}(x, u, v)=0
\end{gathered}
$$

have been established in $[4,11,16,19-21]$. In particular, in [20], the existence of the equilibrium solutions is established for the Volterra-Lotka mutualistic symbiosis model in the case of equal linear birth rates, using the method of upper and lower solutions. However, for so-called mixed type in which $F_{1}$ and $F_{2}$ involve both singular and sublinear terms, results regarding the existence of positive entire solutions cannot be derived from those in the literature.

The aim of this article is to develop the theory of existence of positive solutions for nonlinear elliptic systems. Based on a comparison principle, using the SchauderTychonoff fixed point theorem, we establish one main theorem regarding the existence of entire positive solutions for the system (1.1). Our results are applicable to systems such 
as

$$
\begin{aligned}
& -\Delta u=f_{1}(x) u^{\alpha}+g_{1}(x) u^{-\beta}+h_{1}(x) u^{\gamma} v^{\delta}, \\
& -\Delta v=f_{2}(x) v^{\alpha}+g_{2}(x) v^{-\beta}+h_{2}(x) v^{\gamma} u^{\delta},
\end{aligned}
$$

or

$$
\begin{aligned}
& -\Delta u=f_{1}(x) u^{\alpha}+g_{1}(x) u^{-\beta}+h_{1}(x) u^{\gamma}\left(c_{0}+v\right)^{-\delta}, \\
& -\Delta v=f_{2}(x) v^{\alpha}+g_{2}(x) v^{-\beta}+h_{2}(x) v^{\gamma}\left(c_{0}+u\right)^{-\delta},
\end{aligned}
$$

with $0<\alpha, \beta, \gamma<1, c_{0} \geq 0,0<\delta<1-\gamma$, and some other kinds of systems even more general (see Remark 2.2). Moreover, our method can be used to deal with similar systems on a bounded domain.

\section{Main results}

First, we denote by $\phi$ the function defined on $\mathbb{R}$ :

$$
\phi(t)=1, \quad \text { if } 0 \leq t<1 ; \quad \phi(t)=t^{2-N}, \quad \text { if } t \geq 1 .
$$

A solution $(u(x), v(x))$ for equation system (1.1) is usually called a minimal positive entire solution if both $u(x)$ and $v(x)$ are between two positive constant multiples of function $\phi(|x|)$ in whole $\mathbb{R}^{N}$. This term comes from the fact that no positive solution of $\Delta u \leq 0$ in an exterior domain can decay more rapidly than a constant multiple of $|x|^{2-N}$, see [18].

Theorem 2.1. Suppose that $0<\alpha, \beta, \gamma<1$ are constants and the functions $g_{i}, h_{i}(i=1,2)$, and $P$ satisfy the following conditions:

$(T) f_{i}, g_{i}, h_{i}$ are locally Hölder continuous with exponent $\theta \in(0,1)$ in $\mathbb{R}^{N}$ and

$$
\begin{gathered}
\int_{1}^{+\infty} s^{N-1-\alpha(N-2)} f_{i}^{*}(s) d s<+\infty, \quad f_{i}^{*}(s)=\max _{|x|=s} f_{i}(x), \\
\int_{1}^{+\infty} s^{N-1+\beta(N-2)} g_{i}^{*}(s) d s<+\infty, \quad g_{i}^{*}(s)=\max _{|x|=s} g_{i}(x), \\
\int_{1}^{+\infty} s^{N-1-\gamma(N-2)} h_{i}^{*}(s) P^{*}(s) d s<+\infty, \quad h_{i}^{*}(s)=\max _{|x|=s} h_{i}(x), P^{*}(s)=\max _{|x|=s} P(\phi(|x|)), \\
f_{i *}(s)+g_{i_{*}}(s)+h_{i *}(s) \neq \equiv \quad \text { for } s \geq 0, \\
f_{i_{*}}(s)=\min _{|x|=s} f_{i}(x), \quad g_{i_{*}}(s)=\min _{|x|=s} g_{i}(x) ; \quad h_{i *}(s)=\min _{|x|=s} h_{i}(x) ;
\end{gathered}
$$

(P) $P: \mathbb{R}_{+} \rightarrow \mathbb{R}_{+}$is a continuous differentiable function satisfying that there exists a $\lambda \in$ $(0,1-\gamma)$ such that for all $k \geq 1$ and $c \in\left[k^{-1}, k\right]$,

$$
P(c s) \leq k^{\lambda} P(s), \quad \forall s>0 .
$$


4 Entire positive solution to systems

Then the system (1.1) possesses a positive entire solution $(u, v) \in \mathbb{C}_{\text {loc }}^{2, \theta}\left(\mathbb{R}^{N}\right) \times \mathbb{C}_{\text {loc }}^{2, \theta}\left(\mathbb{R}^{N}\right)$ such that each of $u$ and $v$ decays between two positive constant multiples of $\phi(|x|)$ as $x$ tends to infinity, that is, the solution is minimal.

Remark 2.2. Examples of function $P(s)$ satisfying the condition $(P)$ are

$$
P(s)=\left(c_{0}+s\right)^{-\delta}, \quad\left(c_{0}>0,0<\delta \leq \lambda\right)
$$

as suggested in (1.8),

$$
P(s)=s^{\delta}+s^{-\sigma}, \quad(0<\delta \leq \lambda, 0<\sigma \leq \lambda)
$$

or

$$
P(s)=\frac{s^{\delta}}{\left(c_{0}+s\right)^{\sigma}}, \quad\left(c_{0}>0, \delta>0, \sigma>0,0<\delta+\sigma \leq \lambda\right)
$$

and so on.

\section{Proof of results}

Lemma 3.1. Consider the equation

$$
-\Delta u=f(x) u^{\alpha}+g(x) u^{-\beta}+h(x) u^{\gamma} .
$$

Suppose that $f, g, h$ are nonnegative functions defined on $\mathbb{R}^{N}$, and $0<\alpha, \beta, \gamma<1$ are constants. If $f, g$, $h$ are locally Hölder continuous with exponent $\theta \in(0,1)$ in $\mathbb{R}^{N}$ and

$\left(T^{\prime}\right)$

$$
\begin{gathered}
\int_{1}^{+\infty} s^{N-1-\alpha(N-2)} f^{*}(s) d s<+\infty, \quad f^{*}(s)=\max _{|x|=s} f(x), \\
\int_{1}^{+\infty} s^{N-1+\beta(N-2)} g^{*}(s) d s<+\infty, \quad g^{*}(s)=\max _{|x|=s} g(x), \\
\int_{1}^{+\infty} s^{N-1-\gamma(N-2)} h^{*}(s) d s<+\infty, \quad h^{*}(s)=\max _{|x|=s} h(x), \\
f_{*}(s)+g_{*}(s)+h_{*}(s) \neq \equiv, \quad \text { for } s \geq 0, \\
f_{*}(s)=\min _{|x|=s} f(x), \quad g_{*}(s)=\min _{|x|=s} g(x), \quad h_{*}(s)=\min _{|x|=s} h(x),
\end{gathered}
$$

then (3.1) possesses a unique positive entire solution $u \in \mathbb{C}_{\text {loc }}^{2, \theta}\left(\mathbb{R}^{N}\right)$ such that $u$ decays between two positive constant multiples of $\phi(|x|)$ as $x$ tends to infinity, that is, the solution is minimal.

Lemma 3.2. Suppose that $f: \mathbb{R}^{N} \times \mathbb{R}^{+} \rightarrow \mathbb{R}$ is a continuous function such that one of the following assumptions is satisfied:

$\left(F_{1}\right) s^{-1} f(x, s)$ is strictly decreasing in $s$ for each $x \in \mathbb{R}^{N}$, 
$\left(F_{2}\right) s^{-1} f(x, s)$ is strictly decreasing in $s$ for each $x$ in a subset $\Omega_{0}$ of $\mathbb{R}^{N}$ and both $f(x, s)$ and $s^{-1} f(x, s)$ are nonincreasing in $s$ for all $x$ in the remainder part $\mathbb{R}^{N}-\Omega_{0}$.

Let $w, v \in \mathbb{C}^{2}\left(\mathbb{R}^{N}\right)$ satisfy

(a) $\Delta w+f(x, w) \leq 0 \leq \Delta v+f(x, v)$ in $\mathbb{R}^{N}$,

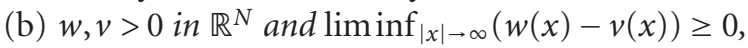

(c) $\Delta v$ in $L^{1}\left(\mathbb{R}^{N}\right)$.

Then $w \geq v \in \mathbb{R}^{N}$.

The proof of Lemma 3.1 is given for completeness in the appendix of this article. Lemma 3.2 is an extension of [17, Lemma 1], so the proof is omitted here for briefness.

Proof of Theorem 2.1. Consider the equation

$$
-\Delta u=f_{1}(x) u^{\alpha}+g_{1}(x) u^{-\beta}+h_{1}(x) u^{\gamma} P(\phi(|x|)), \quad x \in \mathbb{R}^{N} .
$$

In view of $(T)$ and Lemma 3.1, we find that there exists, for (3.3), a unique entire positive solution $u_{0}(x) \in \mathbb{C}_{\text {loc }}^{2, \theta}\left(\mathbb{R}^{N}\right)$. With the same argument, for the equation

$$
-\Delta v=f_{2}(x) v^{\alpha}+g_{2}(x) v^{-\beta}+h_{2}(x) v^{\gamma} P(\phi(|x|)), \quad x \in \mathbb{R}^{N},
$$

there exists a unique entire positive solution $v_{0}(x) \in \mathbb{C}_{\text {loc }}^{2, \theta}\left(\mathbb{R}^{N}\right)$. Moreover, it is obvious that there is a constant $c_{0}>1$ such that for any $x \in \mathbb{R}^{N}$,

$$
\begin{gathered}
c_{0}^{-1} \phi(|x|) \leq u_{0}(x) \leq c_{0} \phi(|x|), \\
c_{0}^{-1} \phi(|x|) \leq v_{0}(x) \leq c_{0} \phi(|x|) .
\end{gathered}
$$

For any constant $E \geq 1$, denote

$$
\begin{gathered}
U_{E} \equiv\left\{u \in \mathbb{C}_{\text {loc }}^{0, \theta}\left(\mathbb{R}^{N}\right) \mid E^{-1} u_{0}(x) \leq u(x) \leq E u_{0}(x), x \in \mathbb{R}^{N}\right\}, \\
V_{E} \equiv\left\{v \in \mathbb{C}_{\operatorname{loc}}^{0, \theta}\left(\mathbb{R}^{N}\right) \mid E^{-1} v_{0}(x) \leq v(x) \leq E v_{0}(x), x \in \mathbb{R}^{N}\right\}, \\
Q \equiv U_{E} \times V_{E} .
\end{gathered}
$$

Obviously, $Q$ is closed and convex. For each $(u, v) \in U_{E} \times V_{E}$, by Poisson equations theory and $(T)$, the problem

$$
-\Delta \tilde{u}=f_{1}(x) u^{\alpha}+g_{1}(x) u^{-\beta}+h_{1}(x) u^{\gamma} P(v), \quad x \in \mathbb{R}^{N},
$$

has a unique solution $\tilde{u} \in \mathbb{C}_{\text {loc }}^{2, \theta}\left(\mathbb{R}^{N}\right) \subset \mathbb{C}_{\text {loc }}^{0, \theta}\left(\mathbb{R}^{N}\right)$, and the problem

$$
-\Delta \widetilde{v}=f_{2}(x) v^{\alpha}+g_{2}(x) v^{-\beta}+h_{2}(x) v^{\gamma} P(u), \quad x \in \mathbb{R}^{N},
$$

has a unique solution $\tilde{v} \in \mathbb{C}_{\text {loc }}^{2, \theta}\left(\mathbb{R}^{N}\right) \subset \mathbb{C}_{\text {loc }}^{0, \theta}\left(\mathbb{R}^{N}\right)$. Defining the mappings $A_{1}: Q \rightarrow \mathbb{C}_{\text {loc }}^{0, \theta}\left(\mathbb{R}^{N}\right)$ by $A_{1}(u, v)=\tilde{u}$ and $A_{2}: Q \rightarrow \mathbb{C}_{\text {loc }}^{0, \theta}\left(\mathbb{R}^{N}\right)$ by $A_{2}(u, v)=\tilde{v}$, we have that $A_{i}(u, v) \in \mathbb{C}_{\text {loc }}^{2, \theta}\left(\mathbb{R}^{N}\right)$, $i=1,2$, and hence

$$
\Phi(Q) \subset \mathbb{C}_{\text {loc }}^{2, \theta}\left(\mathbb{R}^{N}\right) \times \mathbb{C}_{\text {loc }}^{2, \theta}\left(\mathbb{R}^{N}\right)
$$


We claim that if $E$ is a positive constant large enough, then

$$
\begin{array}{ll}
E^{-1} u_{0}(x) \leq \tilde{u}(x) \leq E u_{0}(x), & x \in \mathbb{R}^{N}, \\
E^{-1} v_{0}(x) \leq \tilde{v}(x) \leq E v_{0}(x), & x \in \mathbb{R}^{N},
\end{array}
$$

hence we have $A_{1}(Q) \subset U_{E}$ and $A_{2}(Q) \subset V_{E}$. In fact, we have

$$
\begin{aligned}
& \left(E c_{0}\right)^{-1} \phi(|x|) \leq E^{-1} u_{0}(x) \leq u(x) \leq E u_{0}(x) \leq\left(E c_{0}\right) \phi(|x|), \\
& -\Delta \tilde{u}=f_{1}(x) u^{\alpha}+g_{1}(x) u^{-\beta}+h_{1}(x) u^{\gamma} P(v) \\
& \quad \leq f_{1}(x) E^{\alpha} u_{0}^{\alpha}+g_{1}(x) E^{\beta} u_{0}^{-\beta}+h_{1}(x) u_{0}^{\gamma} E^{\gamma} P\left(\frac{v(x)}{\phi(|x|)} \phi(|x|)\right) \\
& \quad \leq f_{1}(x) E u_{0}^{\alpha}+g_{1}(x) E u_{0}^{-\beta}+h_{1}(x) u_{0}^{\gamma} E^{\gamma}\left(E c_{0}\right)^{\lambda} P(\phi(|x|)),
\end{aligned}
$$

while on the other hand, we have

$$
-\Delta\left(E u_{0}\right)=f_{1}(x) E u_{0}^{\alpha}+g_{1}(x) E u_{0}^{-\beta}+h_{1}(x) u_{0}^{\gamma} E P(\phi(|x|))
$$

Thus, if $E$ is so large that $E^{(1-r-\lambda) / \lambda} \geq c_{0}$, then we have $\Delta \tilde{u} \geq \Delta\left(E u_{0}\right)$. It follows from the maximum principle for the operator $-\Delta$ that

$$
\tilde{u}(x) \leq E u_{0}(x), \quad x \in \mathbb{R}^{N} .
$$

Similarly, we have

$$
\tilde{u}(x) \geq E^{-1} u(x), \quad x \in \mathbb{R}^{N} .
$$

With the same argument, we conclude that

$$
E^{-1} v_{0}(x) \leq \widetilde{v}(x) \leq E v_{0}(x), \quad x \in \mathbb{R}^{N} .
$$

Fix this $E$ and define $\Phi: Q \rightarrow Q$ by

$$
\Phi(u, v)=\left(A_{1}(u, v), A_{2}(u, v)\right), \quad \forall(u, v) \in Q,
$$

and now we only need to prove that $\Phi$ has a fixed point in $Q$.

In order to use the Schauder-Tychonoff fixed point theorem, we will prove that the operator $\Phi$ satisfies the conditions through three steps.

(1) $\Phi(Q) \subset Q$. This is a direct conclusion of (3.10).

(2) $\Phi: Q \rightarrow Q$ is continuous. Obviously, it suffices to prove that $A_{1}$ and $A_{2}$ are both continuous in the sense that for $w_{n} \rightarrow w$ in $Q$, it holds true that $\left\|A_{i} w_{n}-A_{i} w\right\|_{0, \theta} \rightarrow 0$, $n \rightarrow \infty, i=1,2$, here, for any sequence $\left\{u_{n}\right\} \subset \mathbb{C}_{\text {loc }}^{0, \theta}\left(\mathbb{R}^{N}\right)$, by writing $\left\|u_{n}\right\|_{0, \theta} \rightarrow 0, n \rightarrow \infty$, we mean that, for any closed bounded domain $G \subset \mathbb{R}^{N},\left\|u_{n}\right\|_{\mathbb{C}^{0, \theta}(G)} \rightarrow 0, n \rightarrow \infty$. 
Denote

$$
\begin{gathered}
F(x) \equiv f_{1}(x) u^{\alpha}+g_{1}(x) u^{-\beta}+h_{1}(x) u^{\gamma} P(v), \\
F_{n}(x) \equiv f_{1}(x) u_{n}^{\alpha}+g_{1}(x) u_{n}^{-\beta}+h_{1}(x) u_{n}^{\gamma} P\left(v_{n}\right) .
\end{gathered}
$$

We have obviously that

$$
\left\|F_{n}-F\right\|_{0, \theta} \longrightarrow 0, \text { as }\left\|u_{n}-u\right\|_{0, \theta}+\left\|v_{n}-v\right\|_{0, \theta} \longrightarrow 0, \quad n \longrightarrow \infty
$$

By Lemma 3.1, we may let $\tilde{u}$ be the unique solution of the equation $\Delta \tilde{u}=F(x)$ and let $\tilde{u}_{n}$ be the unique solution of the equation $\Delta \tilde{u}_{n}=F_{n}(x)$. Then by the Schauder estimation theory, we know that for any bounded domain $G \subset \mathbb{R}^{N}$, there exists a constant $\mathbb{C}>0$ such that

$$
\left\|\tilde{u}_{n}-\tilde{u}\right\|_{\mathbb{C}^{2, \theta}(\bar{G})} \leq \mathbb{C}\left\|F_{n}-F\right\|_{\mathbb{C}^{0, \theta}(\bar{G})},
$$

and hence

$$
\left\|\tilde{u}_{n}-\tilde{u}\right\|_{\mathbb{C}^{0, \theta}(\bar{G})} \leq \mathbb{C}\left\|F_{n}-F\right\|_{\mathbb{C}^{0, \theta}(\bar{G})} .
$$

Therefore,

$$
\left\|\tilde{u}_{n}-\tilde{u}\right\|_{0, \theta} \longrightarrow 0, \quad \text { as }\left\|u_{n}-u\right\|_{0, \theta}+\left\|v_{n}-v\right\|_{0, \theta} \longrightarrow 0, \quad n \longrightarrow \infty
$$

that is, $A_{1}$ is a continuous mapping from $Q$ to $U_{E}$. Similarly, $A_{2}$ is also a continuous mapping from $Q$ to $V_{E}$.

(3) $\Phi(Q)$ is relatively compact in $\mathbb{C}_{\text {loc }}^{0, \theta}\left(\mathbb{R}^{N}\right) \times \mathbb{C}_{\text {loc }}^{0, \theta}\left(\mathbb{R}^{N}\right)$.

We first recall the gradient estimates for Poisson's equation (see [8]). For any bounded domain $\Omega \subset \mathbb{R}^{N}$, if $\Delta u=f$ in $\Omega$, then

$$
\sup _{\Omega} d_{x}|D u(x)| \leq \mathbb{C}\left(\sup _{\Omega}|u|+\sup _{\Omega} d_{x}^{2}|f(x)|\right),
$$

where $d_{x}=\operatorname{dist}(x, \partial \Omega)$ and $\mathbb{C}=\mathbb{C}(N)$.

Denote $B_{m} \equiv\left\{x \in \mathbb{R}^{N} ;\|x\|<m\right\}, m=1,2, \ldots$ For each $u \in A_{1}(Q)$, we have by (3.22) that

$$
\begin{aligned}
\sup _{B_{m}}|D u(x)| & \leq \sup _{B_{m}} d_{x}|D u(x)| \leq \sup _{B_{m+1}} d_{x}|D u(x)| \\
& \leq \mathbb{C}\left(\sup _{B_{m+1}}|u(x)|+\sup _{B_{m+1}} d_{x}^{2}|F(x)|\right) \\
& \leq \mathbb{C}\left(\sup _{B_{m+1}}|E \phi(x)|+(m+1)^{2} \sup _{B_{m+1}}|F(x)|\right) \leq K_{m}
\end{aligned}
$$

where $K_{m}$ depends only on $m$ and $N$. 
Furthermore, by (3.23), we know that

$$
\frac{|u(x)-u(y)|}{|x-y|} \leq\left|D u\left(t_{0} x+\left(1-t_{0}\right) y\right)\right| \leq K_{m}, \quad \forall x, y \in B_{m} .
$$

This shows that $A_{1}(Q)$, restricted on $\bar{B}_{m}$, is a bounded subset of $\mathbb{C}^{0,1}\left(\bar{B}_{m}\right)$. By the compact embedding result (see $[1]) ; \mathbb{C}^{0,1}(\bar{\Omega}) \hookrightarrow \hookrightarrow \mathbb{C}^{0, \theta}(\bar{\Omega})$, for any bounded domain $\Omega \subset \mathbb{R}^{N}$, it is seen that $A_{1}(Q)$, restricted on $\bar{B}_{m}$, is a relative compact subset of $\mathbb{C}^{0, \theta}\left(\bar{B}_{m}\right)$. Therefore, for any arbitrary sequence $\left\{u_{n}\right\}_{n \geq 1} \subset A_{1}(Q)$, there exists a subsequence $\left\{u_{n}^{(m)}\right\}_{n \geq 1} \subset A_{1}(Q)$ which is convergent on $\bar{B}_{m}$ in the sense of the norm $\|\cdot\|_{\mathbb{C}^{0, \theta}\left(\bar{B}_{m}\right)}$. The case for $A_{2}(Q)$ is similar.

Considering $\bigcup_{m=1}^{\infty} B_{m}=\mathbb{R}^{N}$, by the diagonal method, we conclude, for $i=1$ and $i=2$, respectively, that for an arbitrary sequence $\left\{u_{n}\right\}_{n \geq 1} \subset A_{i}(Q)$, there exists a subsequence, say, $\left\{u_{n}^{(n)}\right\}_{n \geq 1} \subset A_{i}(Q)$, which is convergent in the sense of the norm $\|\cdot\|_{\mathbb{C}^{0, \theta}(K)}$ on any compact subset $K$ of $\mathbb{R}^{N}$, that is, $A_{i}(Q)$ is relatively compact in $\mathbb{C}_{\text {loc }}^{0, \theta}\left(\mathbb{R}^{N}\right)$. Therefore, $\Phi(Q)=A_{1}(Q) \times A_{2}(Q)$ is a relatively compact subset of $\mathbb{C}_{\text {loc }}^{0, \theta}\left(\mathbb{R}^{N}\right) \times \mathbb{C}_{\text {loc }}^{0, \theta}\left(\mathbb{R}^{N}\right)$.

Therefore, by the Schauder-Tychonoff fixed point theorem, there exists an element $(u, v) \in Q$ such that $\Phi(u, v)=(u, v)$, that is, $(u, v)$ satisfies the system (1.1). This completes the proof of Theorem 2.1.

\section{Appendix}

Proof of Lemma 3.1. Let

$$
\begin{aligned}
& F(x, u)=f(x) u^{\alpha}+g(x) u^{-\beta}+h(x) u^{\gamma}, \\
& G(t, u)=f^{*}(t) u^{\alpha}+g^{*}(t) u^{-\beta}+h^{*}(t) u^{\gamma}, \\
& g(t, u)=f_{*}(t) u^{\alpha}+g_{*}(t) u^{-\beta}+h_{*}(t) u^{\gamma},
\end{aligned}
$$

then $g(|x|, u) \leq F(x, u) \leq G(|x|, u), x \in \mathbb{R}^{N}, u>0$. It follows from $\left(T^{\prime}\right)$ that

$$
0<\int_{0}^{+\infty} s^{N-1} g(s, \phi(s)) d s<\int_{0}^{+\infty} s^{N-1} G(s, \phi(s)) d s<+\infty
$$

Then we define two functions by

$$
y(t)=\mathscr{E}[G(t, \phi(t))], \quad z(t)=\mathscr{E}[g(t, \phi(t))], \quad t>0,
$$

where $\mathscr{F}$ is the integral operator defined by

$$
\mathscr{F}[E](t)=\frac{1}{N-2}\left[\int_{0}^{t}\left(\frac{s}{t}\right)^{N-2} s E(s) d s+\int_{t}^{+\infty} s E(s) d s\right], \quad t>0 .
$$


By the simple calculation, we have

$$
\begin{gathered}
y^{\prime \prime}+\frac{N-1}{t} y^{\prime}=-G(t, \phi(t)), \quad z^{\prime \prime}+\frac{N-1}{t} z^{\prime}=-g(t, \phi(t)), \quad t>0, \\
l_{1} \phi(t) \leq y(t), \quad z(t) \leq l_{2} \phi(t), \quad t>0,
\end{gathered}
$$

for some positive constants $l_{1}$ and $l_{2}$.

Take $\lambda=\max \{\alpha, \beta, \gamma\}$, then for any $k \geq 1$, if $k^{-1} \leq c \leq k$, then

$$
\begin{aligned}
& G(t, c u) \leq k^{\lambda} G(t, u), \quad t \geq 0, u>0, \\
& g(t, c u) \geq k^{-\lambda} g(t, u), \quad t \geq 0, u>0 .
\end{aligned}
$$

Moreover, letting $y_{*}(x)=k_{1}^{\lambda} y(x)$, and $k_{1}$ is a number such that $l_{1} k_{1}^{\lambda} \geq 1$, we have

$$
\begin{gathered}
y_{*}^{\prime \prime}+\frac{N-1}{t} y_{*}^{\prime}=-k_{1}^{\lambda} G(t, \phi(t)), \quad t>0, \\
\phi(t) \leq y_{*}(t) \leq k_{1}^{\lambda} l_{2} \phi(t), \quad t>0,
\end{gathered}
$$

by (A.5) and (A.6). Hence,

$$
\begin{aligned}
G\left(t, y_{*}\right) & =f^{*}(t) y_{*}^{\alpha}+g^{*}(t) y_{*}^{-\beta}+h^{*}(t) y_{*}^{\gamma} \\
& \leq f^{*}(t)\left(l_{2} k_{1}^{\lambda}\right)^{\alpha} \phi^{\alpha}+g^{*}(t) \phi^{-\beta}+h^{*}(t)\left(l_{2} k_{1}^{\lambda}\right)^{\gamma} \phi^{\gamma} \\
& \leq k_{1}^{\lambda}\left[f^{*}(t) \phi^{\alpha}+g^{*}(t) \phi^{-\beta}+h^{*}(t) \phi^{\gamma}\right]=k_{1}^{\lambda} G(t, \phi),
\end{aligned}
$$

where we take $k_{1}$ so big that $k_{1} \geq l_{2}^{\alpha / \lambda(1-\alpha)}$ and $k_{1} \geq l_{2}^{\gamma / \lambda(1-\gamma)}$, that is, $\left(l_{2} k_{1}^{\lambda}\right)^{\alpha} \leq k_{1}^{\lambda}$ and $\left(l_{2} k_{1}^{\lambda}\right)^{\gamma} \leq k_{1}^{\lambda}$.

Therefore, it follows that

$$
y_{*}^{\prime \prime}+\frac{N-1}{t} y_{*}^{\prime} \leq-G\left(t, y_{*}\right)
$$

Similarly, letting $z_{*}(x)=k_{2}^{-\lambda} z(x)$ with constant $k_{2}^{-\lambda} l_{2} \leq 1$, we obtain

$$
z_{*}^{\prime \prime}+\frac{N-1}{t} z_{*}^{\prime} \geq-g\left(t, z_{*}\right),
$$

such that $z_{*}(t)<\delta$ for any $t>0$ and

$$
z_{*}(t) \leq y_{*}(t), \quad t>0 .
$$

Define $y_{*}(0)$ and $z_{*}(0)$ by continuity with (A.3), and let $v(x)=y_{*}(|x|)$ and $w(x)=$ $z_{*}(|x|)$ for $x \in \mathbb{R}^{N}$, then from (A.10) and (A.11) $v$ and $w$ are, respectively, a supersolution and a subsolution of (3.1), with $v(x) \geq w(x)$ satisfied. Therefore, by super-subsolution principle, (3.1) has a positive entire solution $u$ such that

$$
w(x) \leq u(x) \leq v(x), \quad x \in \mathbb{R}^{N} .
$$


For proving the uniqueness of such solutions, we suppose that $v$ and $w$ are two positive entire solutions of (3.1), then it is easily seen that the conditions (a) and (b) in Lemma 3.2 are satisfied even when $v$ interchanges with $w$.

Moreover, since $|\Delta v|$ and $|\Delta w|$ are, respectively, given by $f(x, v)$ and $f(x, w)$, and both $v$ and $w$ are between two constant multiples of $\phi(|x|)$, we have, by (A.5) for some $k>0$,

$$
|\Delta v(x)|,|\Delta w(x)| \leq k^{\lambda} G(x, \phi(|x|))
$$

hence it follows from (A.2) that both $\Delta v$ and $\Delta w$ are in $L^{1}\left(\mathbb{R}^{N}\right)$.

Therefore, using Lemma 3.2, we have $v \geq w$ as well as $w \geq v$ in $\mathbb{R}^{N}$, and hence $v \equiv w$.

\section{References}

[1] R. A. Adams, Sobolev Spaces, Academic Press, New York, 1975.

[2] D. Aronson, M. G. Crandall, and L. A. Peletier, Stabilization of solutions of a degenerate nonlinear diffusion problem, Nonlinear Analysis 6 (1982), no. 10, 1001-1022.

[3] A. Callegari and A. Nachman, Some singular, nonlinear differential equations arising in boundary layer theory, Journal of Mathematical Analysis and Applications 64 (1978), no. 1, 96-105.

[4] D. G. de Figueiredo and J. Yang, Decay, symmetry and existence of solutions of semilinear elliptic systems, Nonlinear Analysis, Theory, Methods \& Applications 33 (1998), no. 3, 211-234.

[5] P. de Mottoni, A. Schiaffino, and A. Tesei, Attractivity properties of nonnegative solutions for a class of nonlinear degenerate parabolic problems, Annali di Matematica Pura ed Applicata, Serie Quarta 136 (1984), 35-48.

[6] A. L. Edelson, Entire solutions of singular elliptic equations, Journal of Mathematical Analysis and Applications 139 (1989), no. 2, 523-532.

[7] N. Fukagai, On decaying entire solutions of second order sublinear elliptic equations, Hiroshima Mathematical Journal 14 (1985), no. 3, 551-562.

[8] D. Gilbarg and N. S. Trudinger, Elliptic Partial Differential Equations of Second Order, 2nd ed., Fundamental Principles of Mathematical Sciences, vol. 224, Springer, Berlin, 1983.

[9] M. E. Gurtin and R. C. MacCamy, On the diffusion of biological populations, Mathematical Biosciences 33 (1977), no. 1-2, 35-49.

[10] N. Kawano, On bounded entire solutions of semilinear elliptic equations, Hiroshima Mathematical Journal 14 (1984), no. 1, 125-158.

[11] N. Kawano and T. Kusano, On positive entire solutions of a class of second order semilinear elliptic systems, Mathematische Zeitschrift 186 (1984), no. 3, 287-297.

[12] O. A. Ladyzhenskaya and N. N. Ural'tseva, Linear and Quasilinear Elliptic Equations, Academic Press, New York, 1968.

[13] A. Nachman and A. Callegari, A nonlinear singular boundary value problem in the theory of pseudoplastic fluids, SIAM Journal on Applied Mathematics 38 (1980), no. 2, 275-281.

[14] M. Naito, A note on bounded positive entire solutions of semilinear elliptic equations, Hiroshima Mathematical Journal 14 (1984), no. 1, 211-214.

[15] A. Okubo, Diffusion and Ecological Problems: Mathematical Models, Biomathematics, vol. 10, Springer, Berlin, 1980.

[16] J. Serrin and H. Zou, Existence of positive entire solutions of elliptic Hamiltonian systems, Communications in Partial Differential Equations 23 (1998), no. 3-4, 577-599. 
[17] J. Shi and M. Yao, On a singular nonlinear semilinear elliptic problem, Proceedings of the Royal Society of Edinburgh. Section A. Mathematics 128 (1998), no. 6, 1389-1401.

[18] C. A. Swanson, Extremal positive solutions of semilinear Schrödinger equations, Canadian Mathematical Bulletin 26 (1983), no. 2, 171-178.

[19] T. Teramoto, Existence and nonexistence of positive entire solutions of second order semilinear elliptic systems, Funkcialaj Ekvacioj 42 (1999), no. 2, 241-260.

[20] L. Z. Wang and A. X. Huang, Existence of positive solutions of a kind of reaction-diffusion system, Journal of Xi'an Jiaotong University 36 (2002), no. 2, 211-213.

[21] C. S. Yarur, Existence of continuous and singular ground states for semilinear elliptic systems, Electronic Journal of Differential Equations 1998 (1998), no. 1, 1-27.

Lingyun Qiu: Department of Mathematics, Tianjin University, Tianjin 300072, China

Current address: Liu Hui Center for Applied Mathematics, Nankai University and Tianjin University, Tianjin 300072, China

E-mail address: Qiu@math.purdue.edu

Miaoxin Yao: Department of Mathematics, Tianjin University, Tianjin 300072, China

Current address: Liu Hui Center for Applied Mathematics, Nankai University and Tianjin University, Tianjin 300072, China

E-mail address: miaoxin@hotmail.com 